\title{
Serverless Cloud-based Speed Advisory Application for Connected Vehicles - Case Study
}

\author{
Hsien-Wen Deng \\ Clemson University \\ M Sabbir Salek ( $\nabla$ msalek@clemson.edu ) \\ Clemson University \\ Mizanur Rahman \\ University of Alabama \\ Mashrur Chowdhury \\ Clemson University \\ Mitch Shue \\ Clemson University \\ Amy W. Apon \\ Clemson University
}

\section{Research Article}

Keywords: Amazon web services, connected vehicles, internet of things, serverless architecture, transportation cyber-physical systems

Posted Date: February 28th, 2022

DOI: https://doi.org/10.21203/rs.3.rs-1377325/v1

License: (c) (i) This work is licensed under a Creative Commons Attribution 4.0 International License.

Read Full License 
1 Serverless Cloud-based Speed Advisory Application for Connected Vehicles -

\section{Case Study}

4 Hsien-Wen Deng ${ }^{1}$, M Sabbir Salek ${ }^{2 *}$, Mizanur Rahman ${ }^{3}$, Mashrur Chowdhury ${ }^{4}$, Mitch Shue ${ }^{5}$, and Amy 5 W. Apon ${ }^{6}$

6

7 'M.S., School of Computing, Clemson University, Clemson, SC 29634, USA; Email:

8 hsienwd@clemson.edu

$9 \quad{ }^{2}$ M.S., Glenn Department of Civil Engineering, Clemson University, Clemson, SC 29634, USA; Email:

10 msalek@clemson.edu

$11{ }^{3}$ Ph.D., Department of Civil, Construction \& Environmental Engineering, The University of Alabama,

12 Tuscaloosa, AL 35487, USA; Email: mizan.rahman@ua.edu

$13{ }^{4}$ Ph.D., Glenn Department of Civil Engineering, Clemson University, 216 Lowry Hall, Clemson, SC

14 29634, USA; Email: mac@clemson.edu

15 5 M.S., School of Computing, Clemson University, Clemson, SC 29634, USA; Email: mshue@clemson.edu

16 ' ${ }^{6}$ h.D., School of Computing, Clemson University, Clemson, SC 29634, USA; Email:

17 aapon@clemson.edu

18

$19 *$ Corresponding author 


\section{ABSTRACT}

In this study, we develop a real-time connected vehicle (CV) speed advisory application, which

22 we refer to as "Serverless CloSA", using commercial cloud services and present case studies for a

23 signalized corridor for different roadway traffic conditions. First, we develop a highly scalable serverless

24 cloud computing architecture using Amazon Web Services (AWS) to support the requirements of a real-

25 time CV application. Second, we develop an optimization-based real-time CV speed advisory algorithm

26 that is deployable in the cloud. Third, we develop a cloud-in-the-loop simulation testbed using AWS and

27 an open-source microscopic roadway traffic simulator called Simulation of Urban Mobility (SUMO).

28 Then, we conduct three case studies for three different roadway traffic conditions, i.e., low, medium, and

29 high-density traffic. Our analyses show that Serverless CloSA can reduce the average stopped delays at

30 signalized intersections in a corridor by $77 \%$ while reducing the aggregated risk of collision by $21 \%$

31 compared to the baseline scenario, i.e., no speed advisory for the CVs. Our experiments show an average

32 end-to-end delay of $452 \mathrm{~ms}$, which is well under the $1000 \mathrm{~ms}$ delay threshold of real-time CV mobility

33 applications. Thus, this study also demonstrates the feasibility of deploying a real-time CV mobility

34 application using commercial cloud services.

36 Keywords: Amazon web services, connected vehicles, internet of things, serverless architecture, 37 transportation cyber-physical systems

\section{$\begin{array}{lll}39 & 1 & \text { INTRODUCTION }\end{array}$}

In transportation cyber-physical systems (TCPS), the interaction between cyber and physical

42 systems makes it possible to develop real-time CV applications [1]. However, to develop a real-time

43 feedback-based interaction between cyber systems and physical systems, high-performance computing

44 infrastructure is required to process the heterogeneous data from different sources. While edge or fog 
computing offers a viable solution to deploy real-time applications in a TCPS environment [2], there are

46 issues related to edge computing-based CV application deployments, such as wireless communication

47 range [3] and maintenance costs. The recent evolution of commercial cloud computing services has made

48 it possible to support real-time TCPS applications in the cloud [4]. Additionally, most commercial cloud

49 service providers now offer serverless solutions (e.g., Lambda [5] offered by Amazon Web Services

50 (AWS), Azure Functions [6] offered by Microsoft Azure) that remove the burden of establishing server

51 instances and enable developers to focus primarily on application development, such as CV and Internet

52 of Things (IoT) applications.

53 In a server-based cloud application, the application developers are required to establish server

54 instances (e.g., AWS EC2 [7]) and configure coding platforms in the cloud that will support the

55 application. On the other hand, in a serverless cloud-based application, the application developers do not

56 need to establish the server instances as the computational resources are managed by the cloud itself

57 based on the computing requirement of an application. Thus, serverless cloud is an attractive option to

58 develop highly scalable real-time CV applications [4]. However, deploying a real-time CV application in

59 a serverless cloud requires developing a feasible serverless cloud architecture utilizing the available cloud

60 services as well as developing an algorithm for the CV application that is deployable through the

61 serverless cloud architecture while meeting the latency requirements of a real-time CV application.

62 In this paper, we develop a CV speed advisory application (i.e., an application that provides each

$63 \mathrm{CV}$ with an advised speed that changes dynamically based on various factors, such as the CV's location,

64 surrounding traffic condition and signal phase and timing of the traffic signal at the intersection that the

$65 \mathrm{CV}$ is approaching) using serverless cloud infrastructure with a goal to minimize the stopped delay

66 experienced by CVs while passing through a signalized corridor, i.e., a roadway with traffic signals

67 deployed at its intersections. In this TCPS environment, commercial serverless cloud infrastructure (as

68 cyber systems) interacts with CVs and connected traffic signals (both as parts of physical systems), as

69 shown in Fig. 1. The serverless cloud infrastructure has three types of service: (i) Function as a Service 


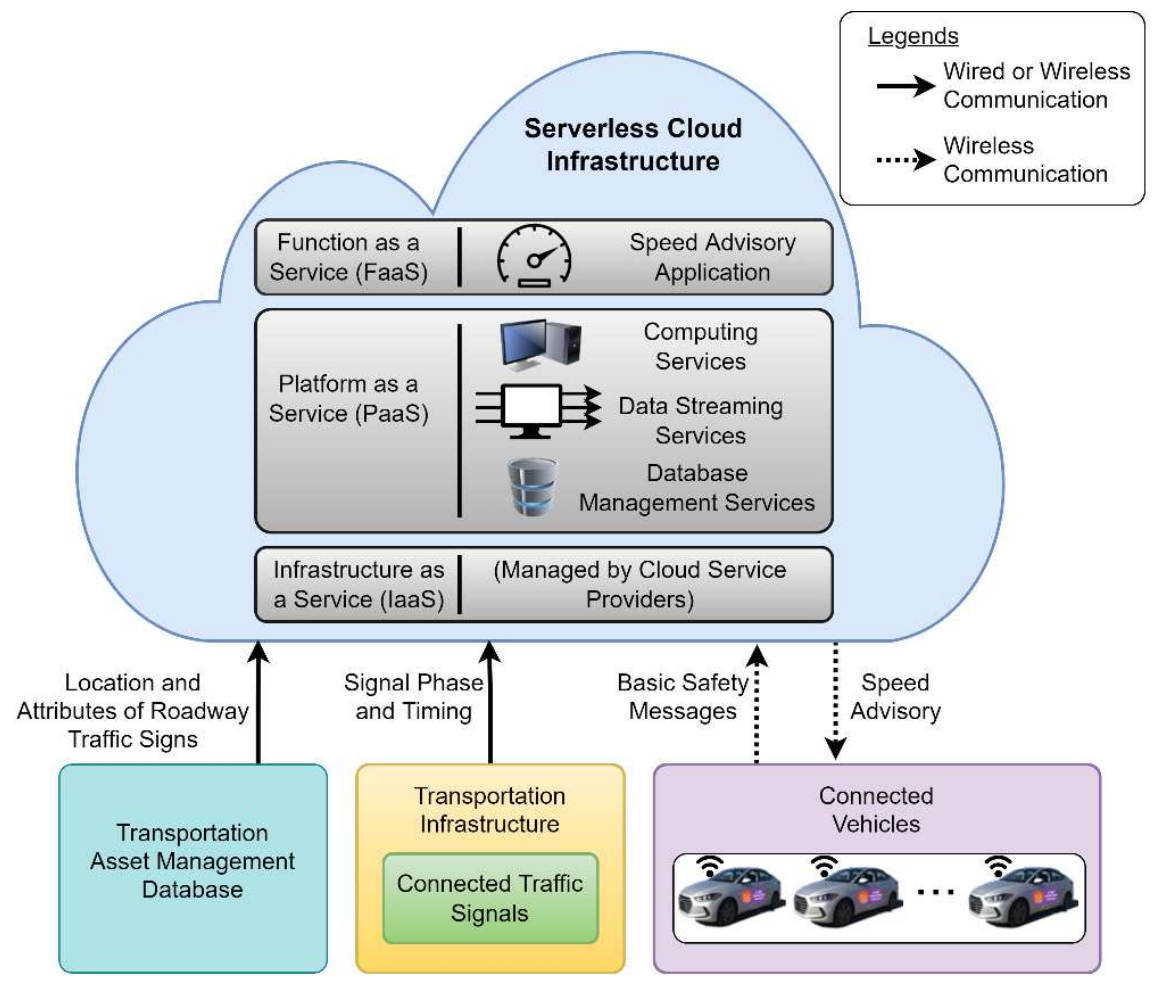

Fig. 1. Serverless cloud architecture of CV speed advisory in TCPS.

70 (FaaS), where a CV application, such as a speed advisory algorithm, can run, (ii) Platform as a Service

71 (PaaS), where computing, data streaming, and database management services operate, and (iii)

72 Infrastructure as a Service (IaaS), which is managed by the cloud service providers in a serverless

73 architecture. The serverless architecture features a pay-as-you-go model without having to manage the

74 underlying computing infrastructure. It is defined as Function as a Service (FaaS), which are serverless

75 functions triggered by events as required by the application $[8,9]$.

The primary contributions of this study are (i) to develop a serverless cloud computing

77 architecture using AWS for a CV speed advisory application in a TCPS environment, which we refer to as

78 "Serverless CloSA" in this paper, and (ii) to develop an optimization-based real-time CV speed advisory

79 application which is deployable in the cloud in terms of end-to-end latency requirement. Similar

80 serverless architectures can also be used to develop other types of CV mobility applications, such as

81 queue warnings and eco-driving advisories, where the maximum end-to-end latency threshold is 
82 considered to be $1000 \mathrm{~ms}$ [10]. In our Serverless CloSA, CVs and connected traffic signals send their

83 state information, i.e., basic safety messages of CVs, and traffic signal phase and timing information of

84 connected traffic signals, into the serverless cloud computing infrastructure. These messages

85 automatically trigger the execution of the serverless functions that support the speed advisory application.

86 Our Serverless CloSA is more scalable in terms of communication coverage area (as CVs directly

87 communicate with the cloud to receive real-time speed advisories) and number of CVs compared to an

88 application supported by traditional edge computing. We also develop a cloud-in-the-loop simulation

89 testbed using AWS and Simulation of Urban Mobility (SUMO) [11], which is a widely used open-source

90 microscopic roadway traffic simulator. Finally, we evaluate the feasibility of Serverless CloSA through a

91 cloud-in-the-loop simulation.

92

\section{RELATED WORK}

94 Cloud infrastructures can effectively communicate with CVs and transportation

95 infrastructures through vehicle-to-infrastructure (V2I) and infrastructure-to-infrastructure (I2I)

96 communication, respectively, using wireless communication technologies, such as Cellular

97 Vehicle-to-Everything (C-V2X), Long-Term Evolution (LTE), and 5G, or wired communication

98 technologies, such as optical fiber-based communication technology. Services in the cloud then

99 aggregate and analyze these data and generate appropriate information corresponding to the

100 cloud applications. For instance, Ning et al. [12] utilized a cloud-based Fog Computing

101 architecture to implement real-time roadway traffic management. Li et. al. [13] provided a

102 maximum value density-based heuristic algorithm through vehicular edge cloud computing to

103 achieve energy usage efficiency for roadway traffic. Jin et. al. [14] presented a method of

104 constructing cloud-based mobility services for connected and automated vehicle (CAV) highway

105 systems. All these studies used a traditional server-based architecture to develop real-time CV 
106 applications. More recently, Deng et al. [4] utilized AWS serverless infrastructure to develop a 107 traffic surveillance application to compute the average speed of CVs in a TCPS environment. 108 However, to our knowledge, no study has used a serverless architecture in a commercial cloud 109 for a real-time $\mathrm{CV}$ application that requires the cloud infrastructure to perform computation 110 using data coming from both CVs and transportation infrastructure in real-time while meeting 111 the strict latency requirement of the CV mobility applications.

112 On the other hand, optimal speed advisory algorithms, that help CVs navigate through a 113 signalized corridor efficiently in terms of reduced stopped delay, fuel consumption, and $\mathrm{CO}_{2}$ 114 emission, have been studied extensively in the literature. Many studies referred to this type of 115 algorithm as the Green Light Optimal Speed Advisory (GLOSA) algorithm [15-20]. For 116 instance, Suzuki and Marumo [18] developed a GLOSA system that projects a green rectangle 117 on the roadway through the head-up display of a GLOSA-enabled vehicle. Stebbins et al. [16] 118 combined model predictive control (MPC) with state-space reduction and GLOSA to yield 119 efficient trajectories for the CVs. However, few studies considered platoon formation in 120 GLOSA. Among them, Stebbins et al. [17] developed a platoon-based optimization technique for 121 GLOSA. The authors included a safety constraint in their optimization model considering that 122 the human drivers may not follow an advised speed if they feel that they will not be able to stop 123 if needed while approaching an intersection. Zhao et al. [21] developed a platoon-based MPC to 124 optimize fuel consumption which enables a platoon of vehicles to pass an intersection within a 125 traffic signal system's green interval, where the model's efficacy was evaluated for different CV 126 penetration rates. However, none of these studies considered a real-time implementation of the 127 platoon-based GLOSA system for speed advisories in a signalized corridor that is "deployable" 128 in a commercial cloud-based TCPS environment. In this study, we develop a platoon-based real- 
129 time CV speed advisory application to minimize the stopped delay experienced by the CVs that 130 is deployable in the commercial clouds in terms of the strict latency requirement of the CV 131 mobility applications.

132

\section{CLOUD-BASED SERVERLESS ARCHITECTURE}

134 AWS maintains a vast cloud infrastructure and services catalog, which makes it secure, 135 scalable, and highly available for developing real-time CV mobility applications [4]. Besides, 136 AWS offers various serverless services, such as AWS Lambda [5], that can be used to develop 137 applications without being concerned about establishing or maintaining any server instances. 138 Such serverless services generally follow pay-as-you-go billing models that make the serverless 139 architectures cost-effective as we mentioned before [22]. Thus, in this study, we develop a 140 serverless cloud-based CV application utilizing the serverless services offered by AWS, such as

141 AWS Lambda. In Fig. 2, we present a serverless cloud architecture showing the computing 142 resources, databases, and streaming services integrated to support a real-time speed advisory 143 application for CVs using AWS. The serverless architecture removes the need for developers to 144 manage traditional server infrastructure. Thus, we only need to focus on developing the 145 application using relevant AWS services. 


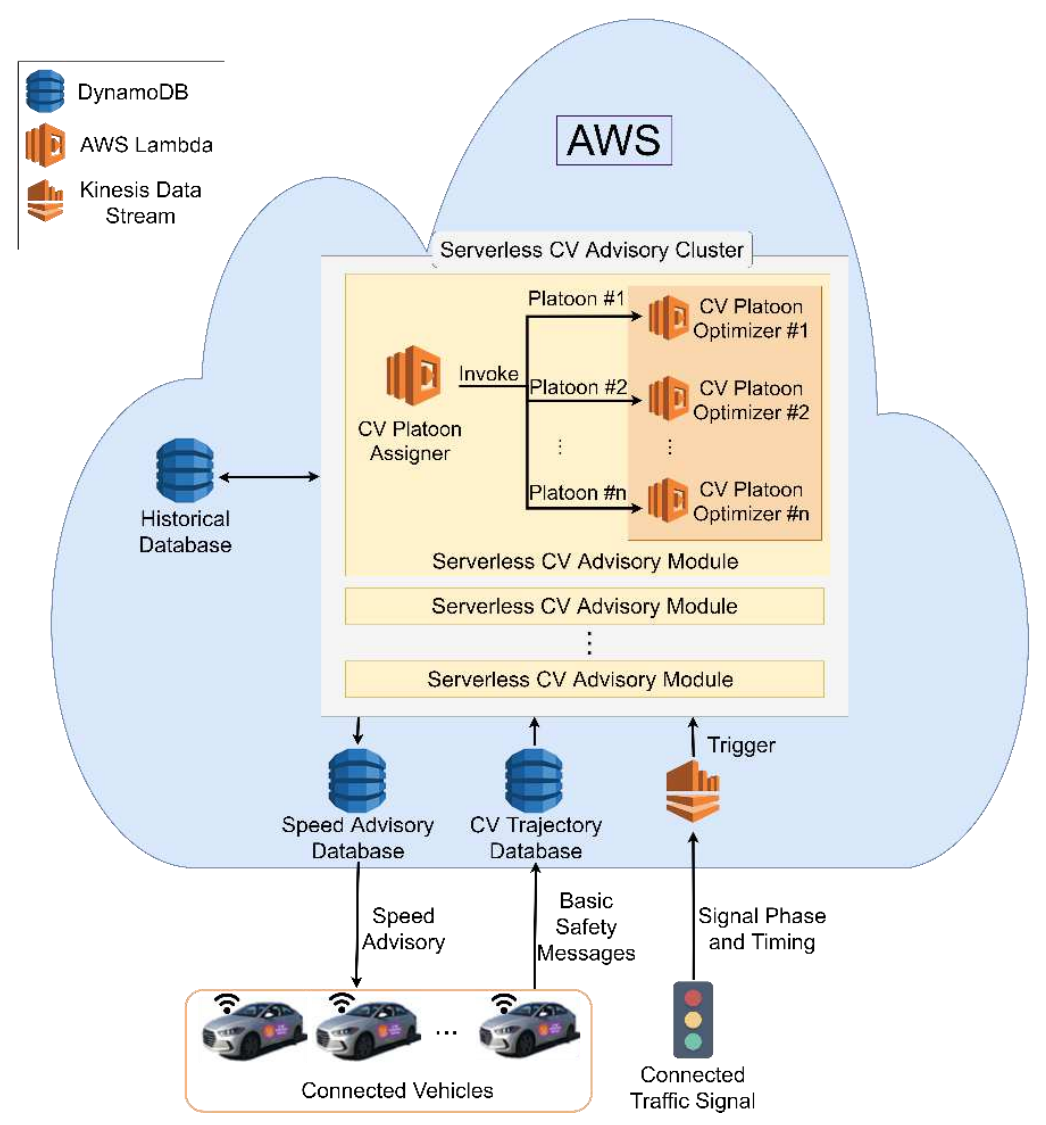

Fig. 2. Details of the Serverless CloSA architecture using AWS services.

148 NoSQL database service with a key-value structure [23], for creating our databases. We create a

149 CV trajectory database to update the CVs' trajectory information, and a speed advisory database

150 to store speed advisory results from which the CVs can download their corresponding speed

151 advisories in real-time. For each traffic signal, we create a historical database to save and update

152 the distances between CVs and the traffic signal in real-time. We utilize Kinesis Data Stream

153 (KDS), a real-time data stream service [24] in AWS, to send a message from each traffic signal

154 to the cloud every second to trigger (i.e., launch the target program automatically) the serverless 
155 functions in CV advisory cluster. AWS Lambda [5] is the serverless compute service at the core

156 of this serverless architecture. We design a group of AWS Lambda functions to form a serverless

157 CV advisory cluster that gets triggered by KDS for each traffic signal. Each cluster contains

158 multiple serverless CV advisory modules that process information from the CVs. To meet the

159 latency requirement of a real-time CV mobility application, i.e., less than or equal to $1000 \mathrm{~ms}$

$160[10,25]$, we define the capacity of each serverless CV advisory module in terms of the maximum

161 number of CVs to be processed, which is $50 \mathrm{CVs}$ per module in our AWS implementation, and

162 run all the $\mathrm{CV}$ advisory modules in parallel. The usage of parallel computing in a cluster makes

163 our Serverless CloSA fast and scalable.

164 There are two types of programs in each serverless CV advisory module: 1) a CV platoon

165 assigner, and 2) a set of CV platoon optimizers. A CV platoon assigner is an AWS Lambda

166 function that has the necessary information related to its corresponding traffic signal and

167 intersection, such as the physical location, signal phase duration of the traffic signal, and the

168 posted speed limit on the roadway approaching that intersection. Once the cluster is triggered,

169 the CV platoon assigner performs the following tasks: 1) collect information from both traffic

170 signals and CVs, 2) split the CVs into platoons based on the CVs' gap information (based on the

171 method discussed in subsection A of section 4), 3) compute a speed advisory for only the leader

172 CV of each platoon (based on the method discussed in subsection 4.2), and 4) save the speed

173 advisory for the leader CV of each platoon into the speed advisory database. Then, for each

174 platoon, the $\mathrm{CV}$ platoon assigner invokes a CV platoon optimizer. A CV platoon optimizer is

175 also a serverless process, i.e., an AWS Lambda, that is responsible for its corresponding CV

176 platoon. It computes speed advisories for the follower CVs in that platoon to help them pass the

177 intersection while maintaining the minimum safety distances and operating within the roadway 
178 speed limit. The results, i.e., the speed advisories for the follower CVs, generated from the CV 179 platoon optimizer are then stored in the speed advisory database.

180 In the real world, each CV generates Basic Safety Messages (BSMs) and each traffic

181 signal generates signal phase and timing messages. In the Serverless CloSA, each CV uploads a 182 filtered BSM including the CV's ID, location, speed, and the gap with its immediate leading CV

183 into the $\mathrm{CV}$ trajectory database. Each traffic signal sends a filtered signal phase and timing 184 message every second containing the current traffic signal phase and the remaining time of that 185 phase through KDS. Our optimization-based speed advisory algorithm deployed in each 186 serverless CV advisory cluster utilizes these BSMs and signal phase and timing messages to 187 generate speed advisories for the CVs in real-time.

1884 CLOUD-BASED SPEED ADVISORY APPLICATION

189 In this section, we present an optimization-based speed advisory application (i.e., 190 Serverless CloSA) running in AWS to minimize the stopped delay for CVs at signalized 191 intersections. The Serverless CloSA consists of three parts: 1) CV platoon identification, 2) 192 optimization-based speed advisory algorithm for the leader CVs of the platoons, and 3) 193 optimization-based speed advisory algorithm for the follower CVs of the platoons. Fig. 3 and 194 Table 1 present all the relevant symbols that we use to develop the application.

\section{4.1 CV Platoon Identification}

196 We form CV platoons based on whether they can pass a signalized intersection within the available time 197 of the current green time or the next green time, i.e., $t_{\text {avail }}(k)$, measured at the $k^{\text {th }}$ timestamp. Therefore, 198 to be identified as a platoon of $N$ number of CVs, the last or $N^{\text {th }} \mathrm{CV}$ of the platoon must be able to pass 199 the intersection within the available time, i.e., meet the following criterion:

$$
\min t_{N, \text { int }}(k) \leq t_{\text {avail }}(k)
$$




Connected
traffic signal

Fig. 3. Relevant symbols related to the speed advisory algorithm.

200 where, $t_{N, \text { int }}(k)$ denotes the estimated time taken by the $N^{\text {th }} \mathrm{CV}$ to reach the intersection from its 201 location at the $k^{\text {th }}$ timestamp. To estimate the minimum of $t_{N, \text { int }}(k)$, we consider the total time required 202 by the $N^{t h} \mathrm{CV}$ to accelerate from its current speed $\left(S_{N}(k)\right)$ to the maximum speed based on the roadway 203 speed limit $\left(S_{\max }\right)$ using its maximum acceleration $\left(a_{A c c}\right)$ and then continue to travel at $S_{\max }$ until it 204 reaches the intersection, which is given by the following equation (according to Newton's equations of 205 motion),

$$
\min t_{N, \text { int }}(k)=\frac{S_{\max }-S_{N}(k)}{a_{A c c}}+\frac{1}{S_{\max }}\left[d_{N, \text { int }}(k)-\frac{\left(S_{\max }\right)^{2}-S_{N}^{2}(k)}{2 a_{A c c}}\right]
$$

First part of the above equation gives the minimum time required by the $N^{\text {th }} \mathrm{CV}$ to accelerate

207 from $S_{N}(k)$ to $S_{\max }$, and the second part of the equation gives the time required by the $N^{\text {th }} \mathrm{CV}$ to reach 208 the intersection at a constant speed (i.e., $S_{\max }$ ) after it achieves $S_{\max }$. Thus, (2) estimates the minimum 209 time required by the $N^{\text {th }} \mathrm{CV}$ of the platoon to reach the intersection. We explain how the time spent in 210 constant speed is obtained for the leader CV of a platoon in the next subsection. 


\begin{tabular}{|c|c|c|c|}
\hline Symbol & Meaning & Symbol & Meaning \\
\hline$\overline{L L}$ & Subscript $L$ refers to the leader CV of a platoon & $d_{i, \text { int }}(k)$ & \multirow{2}{*}{$\begin{array}{l}\text { Distance from the } i^{\text {th }} \text { follower CV in a platoon } \\
\text { to the target intersection at the } k^{\text {th }} \text { timestamp }\end{array}$} \\
\hline$i$ & $\begin{array}{l}\text { Subscript } i \text { refers to the } i^{\text {th }} \text { follower } \mathrm{CV} \text { in a } \\
\text { platoon, i.e., } i \in\{1,2,3, \ldots \ldots N\} \text { for a platoon of } \\
(N+1) \mathrm{CVs} \text { consisting one leader } \mathrm{CV} \text { and } N \\
\text { follower CVs }\end{array}$ & $d_{L, i n t}(k)$ & \\
\hline$x_{L}(k)$ & $\begin{array}{l}\text { Location of the leader } \mathrm{CV} \text { at the } k^{\text {th }} \text { timestamp } \\
\text { with respect to the target intersection }\end{array}$ & $d_{L, \text { constSpd }}(k)$ & $\begin{array}{l}\text { Estimated (at the } k^{t h} \text { timestamp) distance } \\
\text { covered by the leader CV of a platoon while } \\
\text { operating at a target speed }\left(S_{L, \text { tar }}(k)\right) \text { from the } \\
\text { moment it achieves } S_{L, t a r}(k)\end{array}$ \\
\hline$g_{i}(k)$ & $\begin{array}{l}\text { Gap between the } i^{t h} \text { follower CV at the } k^{t h} \\
\text { timestamp with its immediate leading CV }\end{array}$ & $t_{N, \text { int }}(k)$ & $\begin{array}{l}\text { of a platoon to reach the intersection from its } \\
\text { location }\left(x_{N}(k)\right)\end{array}$ \\
\hline$g_{i, t a r}(k)$ & $\begin{array}{l}\text { Target gap of the } i^{t h} \text { follower CV at the } k^{t h} \\
\text { timestamp }\end{array}$ & \multirow[t]{2}{*}{$t_{L, \text { const } A c c}(k)$} & \multirow[t]{2}{*}{$\begin{array}{l}\text { Estimated time required by the leader } \mathrm{CV} \text { of a } \\
\text { platoon from the } k^{t h} \text { timestamp to accelerate } \\
\text { from } S_{L}(k) \text { to a target speed }\left(S_{L, t a r}(k)\right)\end{array}$} \\
\hline$g_{\text {stand }}$ & Constant standstill gap & & \\
\hline & Constant time gap & \multirow[t]{3}{*}{$t_{L, \text { constSpd }}(k)$} & \multirow{3}{*}{$\begin{array}{l}\text { Time required (estimated at the } k^{t h} \text { timestamp) } \\
\text { by the leader CV of a platoon to reach the } \\
\text { intersection while operating at a target speed } \\
\left(S_{L, t a r}(k)\right) \text { from the moment it achieves } \\
S_{L, t a r}(k)\end{array}$} \\
\hline$S_{i}(k)$ & Speed of the $i^{t h}$ follower CV at the $k^{t h}$ timestamp & & \\
\hline$S_{L}(k)$ & $\begin{array}{l}\text { Speed of the leader } \mathrm{CV} \text { of a platoon at the } k^{\text {th }} \\
\text { timestamp }\end{array}$ & & \\
\hline$S_{\max }$ & $\begin{array}{l}\text { Maximum speed, which is same as the roadway } \\
\text { speed limit }\end{array}$ & $t_{\text {avail }}(k)$ & $\begin{array}{l}\text { Available time to pass an intersection calculated } \\
\text { at the } k^{\text {th }} \text { timestamp }\end{array}$ \\
\hline$a_{A c c}$ & Maximum acceleration & $t_{G}$ & (Minimum) green interval \\
\hline$a_{B r k}$ & Maximum braking deceleration & $t_{A R}$ & All red interval \\
\hline$a_{\text {const }}$ & $\begin{array}{l}\text { Constant acceleration; } a_{\text {const }}=a_{A c c} \text { if the } \mathrm{CV} \text { is } \\
\text { accelerating, and } a_{\text {const }}=a_{B r k} \text { if the } \mathrm{CV} \text { is } \\
\text { decelerating }\end{array}$ & \multirow[t]{2}{*}{$t_{Y}$} & \multirow[t]{2}{*}{ Yellow interval } \\
\hline $\operatorname{delay}_{L}(k)$ & $\begin{array}{l}\text { Additional estimated delay calculated from the } k^{t h} \\
\text { timestamp experienced by the leader CV of a } \\
\text { platoon while following } S_{L, a d v}(k) \text { compared to } \\
\text { following } S_{\max }\end{array}$ & & \\
\hline
\end{tabular}


214 two cases to consider based on the current phase of the traffic signal at the target intersection that the CVs

215 are approaching; case I: the platoon can pass the intersection within the current green interval, and case II:

216 the platoon can pass the intersection in the next green interval. For case I, the available time to reach the 217 intersection before the signal turns red is,

$$
t_{\text {avail }}(k)=t_{\text {remain }}(k)
$$

218 where, $t_{\text {remain }}(k)$ is the remaining green interval, whereas, for case II, this available time is an aggregate 219 of the remaining green interval and the other intervals till the next green interval, i.e., sum of the 220 minimum green intervals $\left(\sum t_{G}\right)$ and yellow intervals $\left(\sum t_{Y}\right)$ for the other approaches in the intersection, 221 and sum of the all-red intervals $\left(\sum t_{A R}\right)$;

$$
t_{\text {avail }}(k)=t_{\text {remain }}(k)+\sum t_{G}+\sum t_{Y}+\sum t_{A R}
$$

\section{$222 \quad 4.2$ Speed Advisory for the Leader CVs of the Platoons}

223 For the leader CV of a platoon, the speed advisory is determined based on whether the platoon is a case I

224 platoon or a case II platoon. For the case I platoons, the speed advisory algorithm attempts to assist the $225 \mathrm{CVs}$ to cross the intersection as fast as possible while operating within the roadway speed limit, $S_{\text {max }}$.

226 Therefore, for case I, the leader CVs are simply advised with the roadway speed limit, $S_{\max }$, as the speed 227 advisory. For the case II platoons, the speed advisories for the leader CVs are found through an 228 optimization with an objective to reduce the estimated delay to pass the intersection.

For a case II platoon, our objective function of the optimization for determining the advisory 230 speed for the leader $\mathrm{CV}$ is the estimated delay experienced by the leader CV while traveling from its 231 current state till it reaches the target intersection. In this context, "delay" is estimated as the additional 232 time required by the leader $\mathrm{CV}$ to reach the intersection using the advised speed, $S_{L, a d v}$, compared to the 233 lowest possible time to reach the intersection using the maximum speed, i.e., $S_{\max }$, which is set to be the 
234 same as the speed limit. Thus, the objective function for this optimization is considered as this additional 235 estimated delay for the leader $\mathrm{CV}$, which is given by the following expressions,

$$
\min _{S_{L, a d v}} \operatorname{delay}_{L}(k)
$$

$$
\begin{gathered}
\text { where, } \operatorname{delay}_{L}(k)=\left(t_{L, \text { constAcc }}(k)+t_{L, \text { constSpd }}(k)\right)_{\text {for } S_{L, a d v}}- \\
\left(t_{L, \text { constAcc }}(k)+t_{L, \text { constSpd }}(k)\right)_{\text {for } S_{\text {max }}}
\end{gathered}
$$

$236\left(t_{L, \text { constAcc }}(k)+t_{L, \text { constSpd }}(k)\right)_{\text {for } S_{L, a d v}}$ and $\left(t_{L, \text { constAcc }}(k)+t_{L, \text { constSpd }}(k)\right)_{\text {for } S_{\max }}$ both consist of 237 two periods:

238 1) acceleration period, $t_{L, \text { constAcc }}(k)$ : the time required to accelerate from the leader CV's current speed, $S_{L}(k)$, to $S_{L, a d v}(k)$ or $S_{\max } ;$ and

2) constant speed period, $t_{L, \text { constSpd }}(k)$ : the time required to reach the intersection at a constant speed, $S_{L, a d v}(k)$ or $S_{\max }$, after achieving $S_{L, a d v}(k)$ or $S_{\max }$.

243 followed to estimate the two periods for $S_{\max }$. The required time to accelerate from $S_{L}(k)$ to $S_{L, a d v}(k)$ is 244 given by,

$$
t_{L, \text { constAcc }}(k)=\frac{S_{L, a d v}-S_{L}(k)}{a_{\text {const }}}
$$

245 where, $a_{\text {const }}=a_{A c c}$ if $S_{L, a d v}(k)>S_{L}(k)$, and $a_{\text {const }}=a_{B r k}$ if $\left.S_{L, a d v}(k)<S_{L}(k)\right)$. Then, we estimate

246 the distance covered during the acceleration period. Distance covered while accelerating from $S_{L}(k)$ to $247 S_{L, a d v}(k)$,

$$
d_{L, \text { const Acc }}(k)=\frac{\left(S_{L, a d v}(k)\right)^{2}-S_{L}^{2}(k)}{2 a_{\text {const }}}
$$

To determine $t_{L, \text { constSpd }}(k)$, first, we need to estimate the distance covered (i.e., $\left.d_{L, c o n s t S p d}(k)\right)$

249 while operating at a constant speed, $S_{L, a d v}(k)$, which can be obtained by subtracting $d_{L, c o n s t A c c}(k)$ from 250 the distance of the leader CV from the target intersection (i.e., $d_{L, \text { int }}(k)$ ), 


$$
d_{L, \text { constSpd }}(k)=d_{L, \text { int }}(k)-d_{L, \text { const } A c c}(k)=d_{L, \text { int }}(k)-\frac{\left(s_{L, a d v}(k)\right)^{2}-S_{L}^{2}(k)}{2 a_{\text {const }}}
$$

Now, we can estimate $t_{L, \text { constspd }}(k)$ for $S_{L, a d v}(k)$ as follows,

$$
t_{L, \text { constSpd }}(k)=\frac{d_{L, \text { constSpd }}(k)}{S_{L, a d v}(k)}=\frac{1}{S_{L, a d v}(k)}\left[d_{L, \text { int }}(k)-\frac{\left(S_{L, a d v}(k)\right)^{2}-S_{L}^{2}(k)}{2 a_{\text {const }}}\right]
$$

Similarly, $t_{L, \text { constAcc }}(k)$ and $t_{L, \text { constspd }}(k)$ for $S_{\max }$ can be written as follows,

$$
\begin{gathered}
t_{L, \text { const Acc }}(k)=\frac{S_{\max }-S_{L}(k)}{a_{\text {const }}} \\
t_{L, \text { constSpd }}(k)=\frac{d_{L, \text { constSpd }}(k)}{S_{\max }}=\frac{1}{S_{\max }}\left[d_{L, \text { int }}(k)-\frac{\left(S_{\max }\right)^{2}-S_{L}^{2}(k)}{2 a_{\text {const }}}\right]
\end{gathered}
$$

254 current state until it reaches the target intersection by substituting the terms derived in (7), (10), (11) and 255 (12) into (6),

$$
\operatorname{delay}_{L}(k)=\left(d_{L, \text { int }}(k)+\frac{S_{L}^{2}(k)}{2 a_{\text {const }}}\right)\left[\frac{1}{S_{L, a d v}(k)}-\frac{1}{S_{\text {max }}}\right]-\frac{S_{L, a d v}(k)-S_{\max }}{2 a_{\text {const }}}
$$

For this speed advisory optimization for the case II platoons' leader CV, we consider the 257 following constraint,

$$
\begin{gathered}
S_{\text {max }}-10 \mathrm{mph} \leq S_{L, \text { adv }} \leq U B \\
\text { where, } U B=\left\{\begin{array}{c}
\min \left(S_{\text {max }}, \frac{d_{L, \text { int }}(k)}{t_{\text {avail }}(k)}\right) \text { if } \frac{d_{L, \text { int }}(k)}{t_{\text {avail }}(k)} \geq\left(S_{\text {max }}-10 \mathrm{mph}\right) \\
\left(S_{\text {max }}-10 \mathrm{mph}\right) \text { if } \frac{d_{L, \text { int }}(k)}{t_{\text {avail }}(k)}<\left(S_{\text {max }}-10 \mathrm{mph}\right)
\end{array}\right.
\end{gathered}
$$

This constraint sets lower and upper bounds to the speed advisory for the case II platoons' leader 259 CVs. The lower bound makes sure that the case II platoons' leader CVs are not advised speeds that are 260 too low compared to the roadway speed limit. To ensure this, the lower bound is set to 10 miles per hour 261 (mph) below the roadway speed limit, $S_{\max }$. We chose this threshold to be $10 \mathrm{mph}$ because a threshold 262 less than $10 \mathrm{mph}$, for example, $5 \mathrm{mph}$ below the speed limit) would leave a small window to select the 263 advisory speeds, and a threshold greater than $10 \mathrm{mph}$, for example, $15 \mathrm{mph}$ below the speed limit, might 264 cause selecting advisory speeds that are too low compared to the roadway speed limit. On the other hand, 
265 the upper bound ensures that 1) the advised speeds do not exceed the roadway speed limit, $S_{\max }$, and 2) 266 the leader CVs do not arrive at the intersection early before the signal turns green again.

267 Note that, if $\frac{d_{L, \text { int }}(k)}{t_{\text {avail }}(k)}<\left(S_{\max }-10 \mathrm{mph}\right)$, then the leader CV needs to slow down to a speed that 268 is lower than $\left(S_{\max }-10 \mathrm{mph}\right)$ to reach the intersection before the signal turns green again. However, as 269 we mentioned above, an advised speed lower than $\left(S_{\max }-10 \mathrm{mph}\right)$ may seem too low considering the 270 speed of other vehicles on the roadway and the drivers may not want to or able to follow that. In that case, 271 the leader $\mathrm{CV}$ is advised a speed equal to $\left(S_{\max }-10 \mathrm{mph}\right)$, as this will be the only solution that meets 272 the constraint (14). On the other hand, if $\frac{d_{L, \text { int }}(k)}{t_{\text {avail }}(k)} \geq\left(S_{\max }-10 \mathrm{mph}\right)$, then we set the minimum value 273 between $S_{\max }$ and $\frac{d_{L, i n t}(k)}{t_{\text {avail }}(k)}$ as the upper bound, which leads the optimization to pick a solution that would 274 minimize the delay defined in (13) by allowing the leader CV to operate at a speed within the speed limit 275 so that it can arrive at the intersection when it would turn green again. Thus, the constraint defined in (14)

276 helps to find speed advisory solutions for the leader CVs that would minimize the stopped delay by 277 slowing the CVs down. On the other hand, the objective function defined in (13) pushes the advisory 278 speed solutions toward the speed limit, $S_{\max }$ (note that, $S_{L, a d v}=S_{\max }$ yields delay $(k)=0$ in $(13)$ ) and 279 the optimization determines $S_{L, a d v}$ that is optimum in terms of the above two opposing conditions. As we mentioned before, this part of the algorithm (i.e., subsection 4.2 in this paper) runs in the 281 "CV Platoon Assigner", a serverless process, i.e., AWS Lambda, as shown in Fig. 2. Once the CV 282 Platoon Assigner assigns the CVs into platoons and determines the advisory speeds for the corresponding 283 leader CVs, it saves the results into the Speed Advisory Database. Then, it invokes CV Platoon

284 Optimizers (i.e., one CV Platoon Optimizer for one CV platoon) to run another algorithm of speed 285 advisory optimization for the follower CVs in the platoons, which we explain in the following subsection.

\section{$286 \quad 4.3 \quad$ Speed Advisory for the Follower CVs in the Platoons}

287 While the leader CVs of the platoons are advised speeds to help the CVs quickly pass the 288 intersection (for case I) or to reduce the stopped delay as much as possible (for case II), the follower CVs 
are advised speeds simply to reduce the gap among the follower CVs as much as possible without causing

290 any safety issues, such as increased collision risks compared to the case when the CVs run without any 291 advisory speeds. We do this using a discrete-time linear model predictive control (MPC)-based 292 optimization algorithm that is solved globally to determine the speed advisories for all the follower CVs 293 in each platoon at each time step. In this subsection, we discuss the detailed formulation of the MPC294 based optimization for the follower CVs' speed advisories. Table 1 and Fig. 3 explain the relevant 295 symbols that are used in this formulation.

First, we assume the advised speeds are achievable by the follower CVs in a platoon within a 297 short period of time $\Delta t$ based on the CVs' maximum acceleration, $a_{A c c}$, or deceleration, $a_{B r k}$, 298 capabilities. Then, assuming constant acceleration or deceleration within this short period of time, $\Delta t$, we 299 can write the following equations of motion for the $i^{t h}$ and the $(i+1)^{t h}$ follower CVs in a platoon,

$$
\begin{gathered}
x_{i}(k+1)=x_{i}(k)+\left(\frac{S_{i}(k)+S_{i, a d v}(k)}{2}\right) \Delta t=x_{i}(k)+u_{i}(k) \Delta t \\
\text { where, } u_{i}(k)=\left(\frac{S_{i}(k)+S_{i, a d v}(k)}{2}\right) \\
\text { similarly, } x_{i+1}(k+1)=x_{i+1}(k)+u_{i+1}(k) \Delta t
\end{gathered}
$$

300 Now, we estimate the gap $\left(g_{i+1}(k+1)\right)$ for the $(i+1)^{\text {th }}$ follower CV with its immediate 301 leading follower $\mathrm{CV}$, i.e., the $i^{\text {th }}$ follower $\mathrm{CV}$, as,

$$
g_{i+1}(k+1)=x_{i+1}(k+1)-x_{i}(k+1)-l_{C V}=g_{i+1}(k)+\left[u_{i+1}(k)-u_{i}(k)\right] \Delta t
$$

In this algorithm, we assume that the lengths of all the CVs are the same, i.e., $l_{C V}$ is the same for 303 all the CVs. However, individual CV length can be used as well if the information is available. Note that, 304 (16) stands for the control input that we seek from our MPC-based optimization. Once we obtain the 305 control inputs, we can easily determine the speed advisories for the follower CVs from (16). Now, as (17) 306 is applicable for all the follower CVs in a platoon, we can write it in an augmented matrix form as 307 follows, 


$$
\begin{gathered}
\boldsymbol{G}(k+1)=\boldsymbol{G}(k)+\boldsymbol{B} \boldsymbol{U}(k) \\
\text { where, } \boldsymbol{G}(k+1)=\left[\begin{array}{c}
g_{L}(k+1) \\
g_{1}(k+1) \\
g_{2}(k+1) \\
\cdots \\
g_{N}(k+1)
\end{array}\right]_{(N+1) \times 1}, \boldsymbol{G}(k)=\left[\begin{array}{c}
g_{L}(k) \\
g_{1}(k) \\
g_{2}(k) \\
\cdots \\
g_{N}(k)
\end{array}\right]_{(N+1) \times 1}, \\
\boldsymbol{B}=\left[\begin{array}{ccccc}
0 & 0 & 0 & & 0 \\
-\Delta t & \Delta t & 0 & \cdots & 0 \\
0 & -\Delta t & \Delta t & & 0 \\
\vdots & \vdots & \ddots & \vdots \\
0 & 0 & 0 & \cdots & \Delta t
\end{array}\right]_{(N+1) \times(N+1)}, \boldsymbol{U}(k)=\left[\begin{array}{c}
u_{L}(k) \\
u_{1}(k) \\
u_{2}(k) \\
\cdots \\
u_{N}(k)
\end{array}\right]_{(N+1) \times 1}
\end{gathered}
$$

Note that, although the speed advisory for the leader CV in a platoon is not sought from this

309 MPC-based optimization, we still include the leader CV in (18) because the gap associated with $1^{\text {st }}$

310 follower $\mathrm{CV}$ in a platoon is calculated with respect to the leader CV of that platoon. However, as the

311 leader CV does not have an immediate leading CV, the dynamics of its gap cannot be formulated as in

312 (17). Therefore, all the entries of the first row of $\boldsymbol{B}$ are set to zeros and $g_{L}(k)$ is set to an arbitrary value.

313 Thus, the gap for the leader $\mathrm{CV}, g_{L}(k)$, will remain unchanged over the prediction horizon irrespective of

314 whatever control inputs are chosen and it will not affect our MPC-based optimization.

315 To determine the follower CVs' target gap at each timestamp, we adopt the constant time gap

316 (CTG) policy. In a CTG policy, all the follower CVs in a platoon are expected to maintain a constant time

317 gap with their immediate leading CVs. Besides, in a platooning operation, CTG policy can help to reduce

318 the collision risks by varying the target gap requirement based on the speed of the vehicles. In this study,

319 we consider a two-second constant time gap, i.e., $T_{g}=2$ seconds, with a two-meter standstill gap, i.e.,

$320 g_{\text {stand }}=2$ meters $[26,27]$. A standstill gap is a minimum gap to avoid the chance of collisions that all

321 CVs must maintain, even if they come to a complete stop. Therefore, the target gap for the $(i+1)^{t h}$

322 follower CVs in a platoon $\left(g_{(i+1), t a r}(k+1)\right)$ can be written as,

$$
g_{(i+1), \operatorname{tar}}(k+1)=S_{i+1}(k) \times T_{g}+g_{\text {stand }}
$$

323 where, $g_{\text {stand }}$ denotes constant standstill distance. As (19) can be written for all the follower CVs in a 
324 platoon, we can write them in an augmented form as follows,

$$
\boldsymbol{G}_{\boldsymbol{t a r}}(k+1)=\boldsymbol{G}_{\boldsymbol{t a r}}(k)
$$

$$
\text { where, } \boldsymbol{G}_{\boldsymbol{t a r}}(k+1)=\left[\begin{array}{c}
g_{L, \operatorname{tar}}(k+1) \\
g_{1, \operatorname{tar}}(k+1) \\
g_{2, \operatorname{tar}}(k+1) \\
\ldots \\
g_{N, \operatorname{tar}}(k+1)
\end{array}\right]_{(N+1) \times 1} \text {, and } \boldsymbol{G}_{\boldsymbol{t a r}}(k)=\left[\begin{array}{c}
S_{L}(k) \times T_{g}+g_{\text {stand }} \\
S_{1}(k) \times T_{g}+g_{\text {stand }} \\
S_{2}(k) \times T_{g}+g_{\text {stand }} \\
\cdots \\
S_{N}(k) \times T_{g}+g_{\text {stand }}
\end{array}\right]_{(N+1) \times 1}
$$

Now, we augment (18) and (20) to get the state dynamics for our MPC-based optimization,

$$
\left[\begin{array}{c}
\boldsymbol{G}(k+1) \\
\boldsymbol{G}_{\boldsymbol{t a r}}(k+1)
\end{array}\right]=\left[\begin{array}{c}
\boldsymbol{G}(k) \\
\boldsymbol{G}_{\boldsymbol{t a r}}(k)
\end{array}\right]+\left[\begin{array}{c}
\boldsymbol{B} \\
\mathbf{0}_{(N+1) \times(N+1)}
\end{array}\right] \boldsymbol{U}(k)
$$

327 rewrite $(21)$ as,

$$
\boldsymbol{X}_{\boldsymbol{a}}(k+1)=\boldsymbol{A}_{\boldsymbol{a}} \boldsymbol{X}_{\boldsymbol{a}}(k)+\boldsymbol{B}_{\boldsymbol{a}} \boldsymbol{U}(k)
$$

where, $\boldsymbol{X}_{\boldsymbol{a}}(k+1)=\left[\begin{array}{c}\boldsymbol{G}(k+1) \\ \boldsymbol{G}_{\boldsymbol{t a r}}(k+1)\end{array}\right], \boldsymbol{X}_{\boldsymbol{a}}(k)=\left[\begin{array}{c}\boldsymbol{G}(k) \\ \boldsymbol{G}_{\boldsymbol{t a r}}(k)\end{array}\right], \boldsymbol{A}_{\boldsymbol{a}}=\boldsymbol{I}_{2(N+1) \times 2(N+1)}$, and

$$
\boldsymbol{B}_{\boldsymbol{a}}=\left[\begin{array}{c}
\boldsymbol{B} \\
\mathbf{0}_{(N+1) \times(N+1)}
\end{array}\right]
$$

where, $\boldsymbol{I}_{2(N+1) \times 2(N+1)}$ is an $(N+1) \times(N+1)$ dimensional identity matrix. As with this MPCpolicy, we define our measured variable as follows,

$$
\boldsymbol{Y}_{\boldsymbol{a}}(k)=\boldsymbol{G}(k)-\boldsymbol{G}_{\boldsymbol{t a r}}(k)=\left[\begin{array}{ll}
\boldsymbol{I}_{(N+1) \times(N+1)} & -\boldsymbol{I}_{(N+1) \times(N+1)}
\end{array}\right]\left[\begin{array}{c}
\boldsymbol{G}(k) \\
\boldsymbol{G}_{\boldsymbol{t a r}}(k)
\end{array}\right]
$$

331 which can be rewritten as,

$$
\boldsymbol{Y}_{\boldsymbol{a}}(k)=\boldsymbol{C}_{\boldsymbol{a}} \boldsymbol{X}_{\boldsymbol{a}}(k)
$$

$$
\text { where, } \boldsymbol{C}_{\boldsymbol{a}}=\left[\boldsymbol{I}_{(N+1) \times(N+1)} \quad-\boldsymbol{I}_{(N+1) \times(N+1)}\right]
$$

333 function as our aim is to minimize the difference between the current gap, $\boldsymbol{G}(k)$, and the target gap,

$334 \boldsymbol{G}_{\boldsymbol{t a r}}(k)$, based on the CTG policy through the speed advisories. Therefore, the cost function for a single- 
335 step prediction horizon (as only one step is required to be predicted based on the state dynamics defined 336 in (22)) can be written as,

$$
\boldsymbol{J}=\boldsymbol{Y}_{\boldsymbol{a}}^{T}(k) \boldsymbol{Y}_{\boldsymbol{a}}(k)
$$

Substituting $\boldsymbol{Y}_{\boldsymbol{a}}(k)$ from (24) into (25), we get,

$$
\begin{gathered}
\boldsymbol{J}=\boldsymbol{X}_{\boldsymbol{a}}^{\boldsymbol{T}}(k) \boldsymbol{C}_{\boldsymbol{a}}^{\boldsymbol{T}} \boldsymbol{C}_{\boldsymbol{a}} \boldsymbol{X}_{\boldsymbol{a}}(k)=\boldsymbol{X}_{\boldsymbol{a}}^{\boldsymbol{T}}(k) \boldsymbol{P} \boldsymbol{X}_{\boldsymbol{a}}(k) \\
\text { where, } \boldsymbol{P}=\boldsymbol{C}_{\boldsymbol{a}}^{\boldsymbol{T}} \boldsymbol{C}_{\boldsymbol{a}}
\end{gathered}
$$

Now, we move on to the constraints for this MPC-based optimization. In this case, we introduce constraints for the control inputs defined in (16) and the measured variables defined in (24). First, the

340 follower CVs should never be advised with speeds that exceed the roadway speed limit, $S_{\max }$, nor should

341 they be advised negative speeds, which leads us to the following constraint,

$$
0 \leq S_{i, a d v}(k) \leq S_{\max }
$$

As each control input is defined as the average of each follower CV's current speed, $S_{i}(k)$, and 343 advised speed, $S_{i, a d v}(k)$, in (16), we can rewrite (27) in terms of the control input as follows,

$$
\frac{S_{i}(k)}{2} \leq u_{i}(k) \leq \frac{1}{2}\left(S_{i}(k)+S_{\max }\right)
$$

Second, as mentioned before, we assume that the advised speeds are achievable by the follower

345 CVs based on their maximum acceleration, $a_{A c c}$, or deceleration, $a_{B r k}$, capabilities. Therefore, we also 346 have,

$$
S_{i}(k)+a_{B r k} \Delta t \leq S_{i, a d v}(k) \leq S_{i}(k)+a_{A c c} \Delta t
$$

Again, we can rewrite (29) in terms of the control input for the $i^{\text {th }}$ follower $\mathrm{CV}$ as,

$$
S_{i}(k)+\frac{1}{2} a_{B r k} \Delta t \leq u_{i}(k) \leq S_{i}(k)+\frac{1}{2} a_{A c c} \Delta t
$$




$$
\max \left(\frac{S_{i}(k)}{2},\left(S_{i}(k)+\frac{1}{2} a_{B r k} \Delta t\right)\right) \leq u_{i}(k) \leq \min \left(\frac{1}{2}\left(S_{i}(k)+S_{\max }\right),\left(S_{i}(k)+\frac{1}{2} a_{A c c} \Delta t\right)\right)
$$

We can write (31) into an augmented form as,

$$
\begin{gathered}
\boldsymbol{U}_{\text {low }}(k) \leq \boldsymbol{U}(k) \leq \boldsymbol{U}_{\text {high }}(k) \\
\text { where, } \boldsymbol{U}_{\text {low }}(k)=\left[\begin{array}{c}
\max \left(\frac{S_{L}(k)}{2},\left(S_{L}(k)+\frac{1}{2} a_{B r k} \Delta t\right)\right) \\
\max \left(\frac{S_{1}(k)}{2},\left(S_{1}(k)+\frac{1}{2} a_{B r k} \Delta t\right)\right) \\
\max \left(\frac{S_{2}(k)}{2},\left(S_{2}(k)+\frac{1}{2} a_{B r k} \Delta t\right)\right) \\
\ldots \\
\max \left(\frac{S_{N}(k)}{2},\left(S_{N}(k)+\frac{1}{2} a_{B r k} \Delta t\right)\right)
\end{array}\right], \text { and } \\
\boldsymbol{U}_{\text {high }}(k)=\left[\begin{array}{c}
\min \left(\frac{1}{2}\left(S_{L}(k)+S^{\text {max }}\right),\left(S_{L}(k)+\frac{1}{2} a_{A c c} \Delta t\right)\right) \\
\min \left(\frac{1}{2}\left(S_{1}(k)+S^{\text {max }}\right),\left(S_{1}(k)+\frac{1}{2} a_{A c c} \Delta t\right)\right) \\
\min \left(\frac{1}{2}\left(S_{2}(k)+S^{\text {max }}\right),\left(S_{2}(k)+\frac{1}{2} a_{A c c} \Delta t\right)\right) \\
\ldots \\
\min \left(\frac{1}{2}\left(S_{N}(k)+S^{\text {max }}\right),\left(S_{N}(k)+\frac{1}{2} a_{A c c} \Delta t\right)\right)
\end{array}\right]
\end{gathered}
$$

Next, we introduce a lower bound for the measured variable, $\boldsymbol{Y}_{\boldsymbol{a}}(k)$, due to safety considerations.

As the optimized solution should not result in a situation where any of the follower CVs has a lower gap than its corresponding target gap based on the CTG policy, we write,

$$
\boldsymbol{Y}_{\boldsymbol{a}}(k) \geq \mathbf{0}_{(N+1) \times 1}
$$

Now, we have all the necessary equations formulated that we need for our MPC-based speed advisory optimization for the follower CVs in a platoon. As our linear MPC formulation includes a quadratic cost function (as given in (26)), we utilize a Python-based open-source solver, i.e., CVXOPT [28], for solving quadratic programming problems to run this MPC-based optimization. As mentioned before, this part of the Serverless CloSA, i.e., subsection 4.3, runs in the CV Platoon Optimizer shown in 


\section{CASE STUDY}

We conduct three case studies for different traffic conditions by developing a cloud-in-

365 the-loop simulation testbed to evaluate the feasibility of the Serverless CloSA at a system level.

366 In addition, we compare the results obtained from the simulation with and without the Serverless

367 CloSA to evaluate the performance improvement in terms of stopped delay of the CVs at the

368 signalized intersections, total travel time of the CVs through the signalized roadway section, and

369 an aggregated collision risk indicator. We also evaluate the communication and processing

370 delays for running the Serverless CloSA application to evaluate the feasibility of our cloud-based

371 speed advisory application in terms of latency requirement of this CV mobility application.

\section{$372 \quad 5.1 \quad$ Cloud-in-the-loop Simulation}

373 We use an open-source microscopic traffic simulator called Simulation Urban Mobility

374 (SUMO) [11] to simulate a section of a roadway including traffic signals and CVs operating in

375 the roadway section. In our cloud-in-the-loop simulation, AWS services (residing in the cloud)

376 are integrated with SUMO (running in a local machine) to evaluate the Serverless CloSA (as

377 shown in Fig. 4). Traffic Control Interface (TraCI) [29] is a Python-based interface compatible

378 with SUMO. As Fig. 4 shows, we use TraCI to extract BSMs (e.g., CVs' location and motion

379 information) and signal phase and timing messages (e.g., current signal interval, remaining green

380 time) from the CVs and the traffic signals, respectively. Data collected from the simulation are

381 packaged and transferred to the AWS cloud through different AWS services, i.e., DynamoDB

382 and KDS, via LTE communication. In the cloud, each KDS triggers a Serverless CV Advisory

383 Cluster, as mentioned before. Each Serverless CV advisory Cluster gets CV trajectory

384 information from the CV Trajectory Database. Each Serverless CV Advisory Cluster also

385 collects and updates the distances of the CVs from its corresponding traffic signal (as shown in 


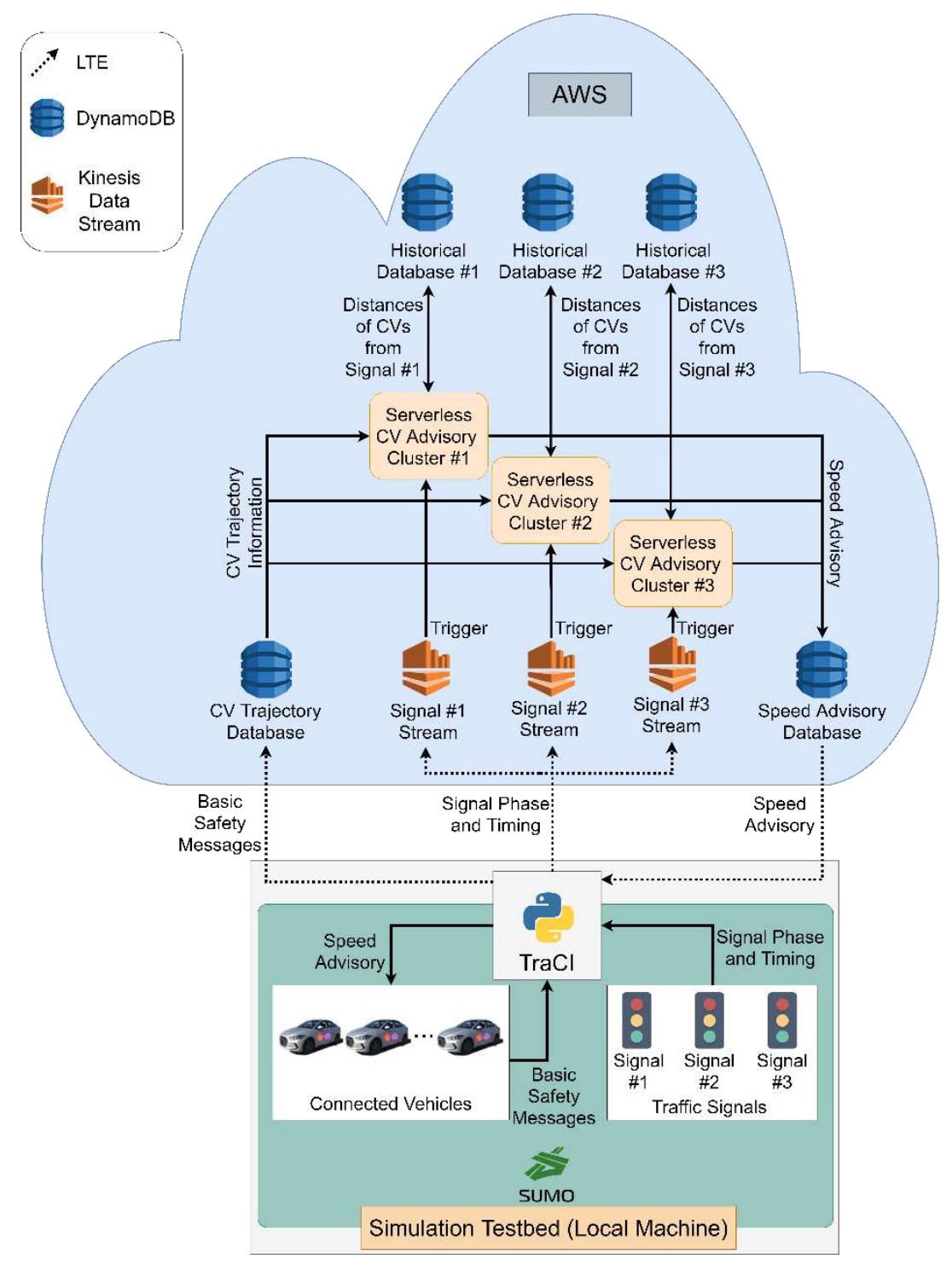

Fig. 4. Dataflow in the cloud-in-the-loop simulation.

386 Fig. 4). Inside the clusters, CV platoon identification and speed advisory optimization algorithms

387 run using serverless processes (details are mentioned in section 3) and the results of the

388 optimizations, i.e., the speed advisories) are saved in the Speed Advisory Database. Then,

389 SUMO can collect the speed advisories via LTE and assign the speed advisories to the CVs

390 through TraCI. 


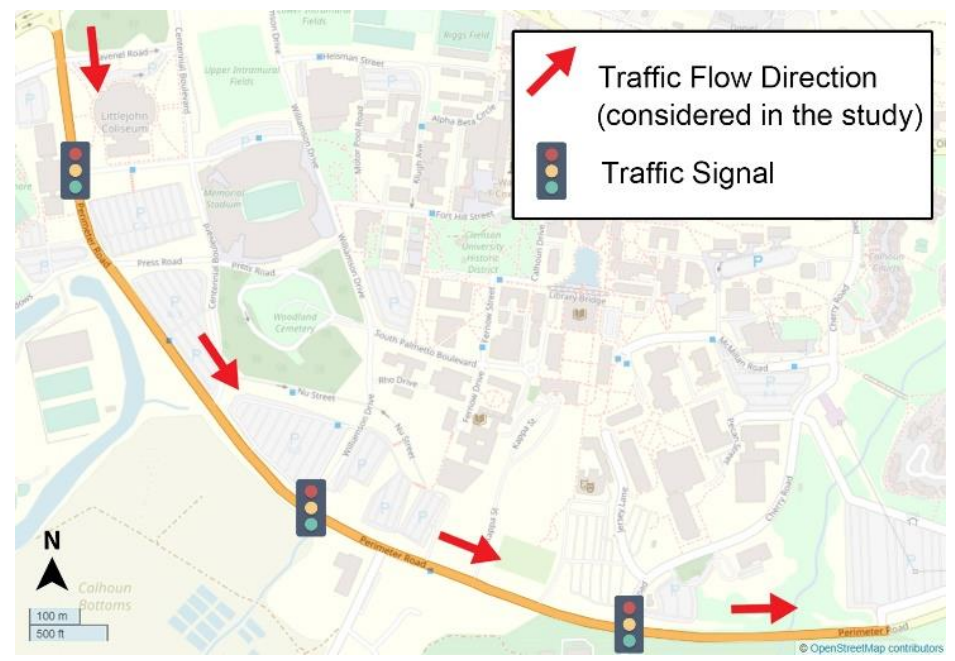

Fig. 5. Route location and layout.

In Fig. 5, the simulated roadway is shown in orange-colored line, which is a 1.5-mile-

392 long 4-lane highway (2 lanes in each direction) with three traffic signals in Clemson, South

393 Carolina, and it is a part of a CV deployment site known as South Carolina Connected Vehicle

394 Testbed (SC-CVT) [30]. By defining the traffic flows in SUMO configuration [31], we generated

$39550 \mathrm{CVs}$ on the simulated roadway in three different traffic densities, i.e., low, medium, and high

396 traffic densities. SUMO allows controlling the time interval within which a given number of

397 vehicles will be generated, which we use here to create the different traffic densities. Here, low

398 traffic density stands for 633 passenger cars per hour per lane (pc/h/ln), which is $33 \%$ of the

399 traffic capacity, i.e., $1900 \mathrm{pc} / \mathrm{h} / \mathrm{ln}$, medium traffic density stands for $1267 \mathrm{pc} / \mathrm{h} / \mathrm{ln}$, i.e., $66 \%$ of

400 traffic capacity, and high traffic density stands for $1900 \mathrm{pc} / \mathrm{h} / \mathrm{ln}$, i.e., full traffic capacity, based

401 on the base saturation flowrate defined in [32]. All CVs operate within a roadway speed limit of

$40235 \mathrm{mph}$, which is already included in the map data. For each condition, we evaluate two

403 scenarios in the simulation: 1) the baseline scenario, i.e., no speed advisory, and 2) the Serverless

404 CloSA-deployed scenario. For each traffic density defined above, we run the simulation five 405 times with randomly generated CVs. 


\subsection{Evaluation Results and Discussions}

To evaluate Serverless CloSA's performance, we compare three measures of

408 effectiveness (MoEs): 1) stopped delay at the signalized intersections of the simulated roadway,

409 2) total travel time to pass the simulated roadway section, and 3) time-integrated time-to-

410 collision (TTC) or TIT. Stopped delay and travel time are MoEs related to traffic flow, whereas

411 TIT is a widely used surrogate measure for evaluating collision risks that integrates the TTC 412 profile below a predefined threshold (i.e., TTC threshold, $T T C^{*}$ ) over time for all the CVs under

413 collision risk evaluation. Details for calculating TIT can be found in [33, 34]. For our study, TIT

414 for the $i^{\text {th }} \mathrm{CV}$ (i.e., TIT $_{i}$ ) can be calculated using the following equation,

$$
\begin{gathered}
\operatorname{TIT}_{i}=\sum_{t}\left[T T C^{*}-T T C_{i}(t)\right], \quad \forall 0 \leq T T C_{i}(t) \leq T T C^{*} \\
\text { where, } T T C_{i}(t)=\left\{\begin{array}{cc}
\frac{g_{i}(t)}{S_{i}(t)-S_{i-1}(t)} & \text { if } S_{i}(t)>S_{i-1}(t) \\
\infty & \text { if } S_{i}(t) \leq S_{i-1}(t)
\end{array}\right.
\end{gathered}
$$

415 Here, $t$ represents a timestamp, $g_{i}(t)$ represents the gap between the $i^{t h} \mathrm{CV}$ (i.e., a follower CV)

416 and the $(i-1)^{t h} \mathrm{CV}$ (i.e., immediate leading $\mathrm{CV}$ of the $\left.i^{t h} \mathrm{CV}\right)$ at $t$, and $S_{i}(t)$ and $S_{i-1}(t)$ represent the

417 speeds of the $i^{t h}$ and the $(i-1)^{t h}$ CVs at $t$, respectively. As observed from (34) and (35), the risk of

418 collision is only considered when the follower CV has a higher speed compared to its immediate leading

419 CV. Once TIT for all the CVs is calculated using (35), we sum them up to get the aggregated TIT for all 420 the CVs within the simulation run time. In this paper, we use a TTC ${ }^{*}$ of 2 seconds based on the time 421 headway requirement in our MPC-based optimization for determining the advisory speeds of the follower 422 CVs. A TTC* of 2 seconds means that whenever the time gap between any two successive CVs is 423 measured to be less than or equal to 2 seconds, the risk of collision is considered in calculating TIT.

Fig. 6 shows box chart comparisons between our Serverless CloSA and the baseline "no speed advisory" scenario in terms of stopped delay and total travel time for three different traffic conditions, i.e., 


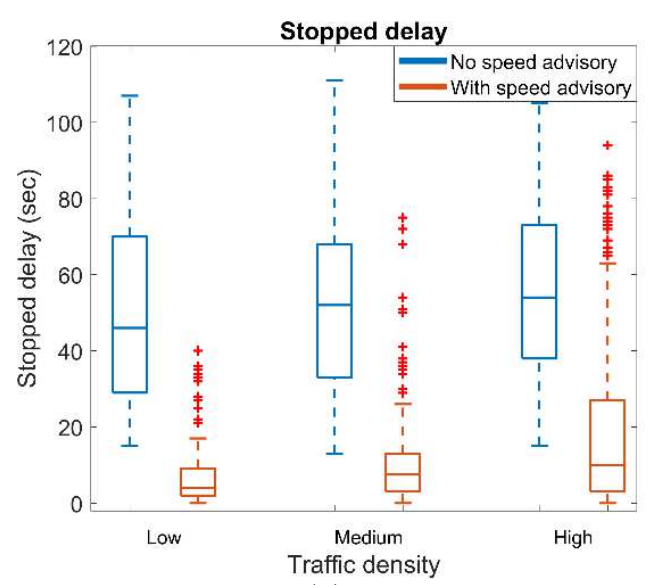

(a)

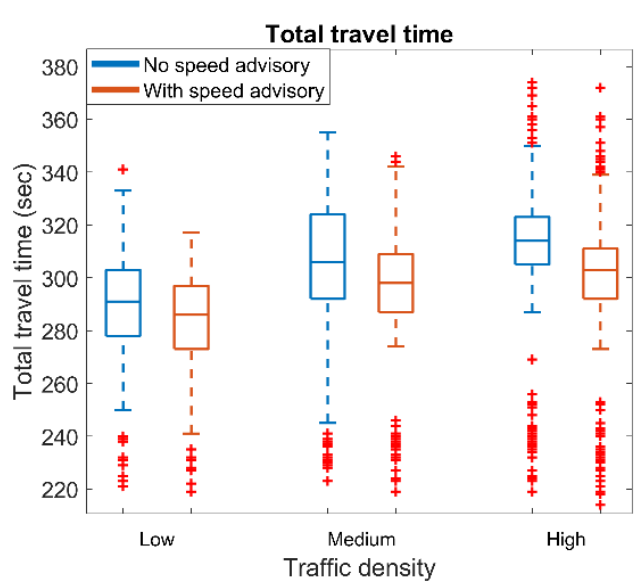

(b)

Fig. 6. Box chart comparisons for (a) stopped delay and (b) total travel time.

427 delay significantly for all three roadway traffic conditions, i.e., low, medium and high-density roadway 428 traffic, compared to the baseline "no speed advisory" scenario. In Fig. 6(b), we observe a small reduction 429 in the total travel time when using Serverless CloSA for providing speed advisories to the CVs as 430 compared to the "no speed advisory" case. This is not unexpected because our speed advisory 431 optimization aims to reduce the stopped delay, not the travel time. While it may seem that a reduction in 432 the stopped delay should cause a reduction in the travel time as well, it may not be the case all the time 433 [35]. For example, note that although the Serverless CloSA reduces the total stopped delay for the CVs 434 significantly, it cannot entirely remove the stopped delay and the CVs may have to stop at the 435 intersections for some time. Then, these CVs would have to start from a stopped condition when the 436 signal turns green again in which case the benefit of having no startup lost time is not achievable. Also, 437 our Serverless CloSA does not advise CVs with speeds considering that they can pass the intersection 438 within the yellow interval. On the other hand, in the "no speed advisory" case, the CVs have no such 439 conditions imposed on them. Thus, reducing the total travel time is not always guaranteed for all the CVs 440 while using Serverless CloSA.

Table 2 presents the effectiveness of Serverless CloSA in terms of percentage reduction of the 442 MoEs on average for each CV in the simulation. We observe that the maximum reduction of the stopped 
Table 2. Average (per CV) reduction of the MOEs for Serverless CloSA

\begin{tabular}{lcccc}
\hline \hline & Low & $\begin{array}{c}\text { Traffic Density } \\
\text { Medium }\end{array}$ & High & $\begin{array}{c}\text { Average of Low, } \\
\text { Medium, and High } \\
\text { Traffic Densities }\end{array}$ \\
\hline \hline Average reduction in stopped delay & $85 \%$ & $80 \%$ & $65 \%$ & $77 \%$ \\
Average reduction in total travel time & $2 \%$ & $3 \%$ & $4 \%$ & $3 \%$ \\
Average reduction in TIT & $24 \%$ & $16 \%$ & $23 \%$ & $21 \%$ \\
\hline \hline
\end{tabular}

443 delay, i.e., about $85 \%$, was possible for low traffic density. In terms of reducing the total travel time,

444 Serverless CloSA's performance did not vary much based on the different traffic conditions. We also

445 observe that Serverless CloSA is most effective in reducing the average per CV TIT, i.e., about $24 \%$, for

446 low-density traffic condition.

447 We also evaluate the end-to-end delay to assess the feasibility of the Serverless CloSA as a real-

448 time CV application. The end-to-end delay is calculated using the following equation,

$$
\text { end-to-end delay }=\text { upload delay }+ \text { processing delay }+ \text { download delay }
$$

$449 \quad$ Fig. 7 presents the processing time, and the end-to-end delay reported during our experiments

450 using box charts and Table 3 provides the averages of the processing time and the end-to-end delay for 451 each CV in the cloud for the three traffic density conditions. From Table 3, the end-to-end delay is about $452452 \mathrm{~ms}$ (on average for all three traffic density conditions), which meets the requirement of a real-time 453 CV mobility application, i.e., maximum allowable delay of $1000 \mathrm{~ms}[10,25]$. Besides, we observe from 454 Fig. 7 and Table 3 that the processing delays and the end-to-end delays do not vary much across the 455 various traffic densities, which indicates the scalability of the Serverless CloSA. 


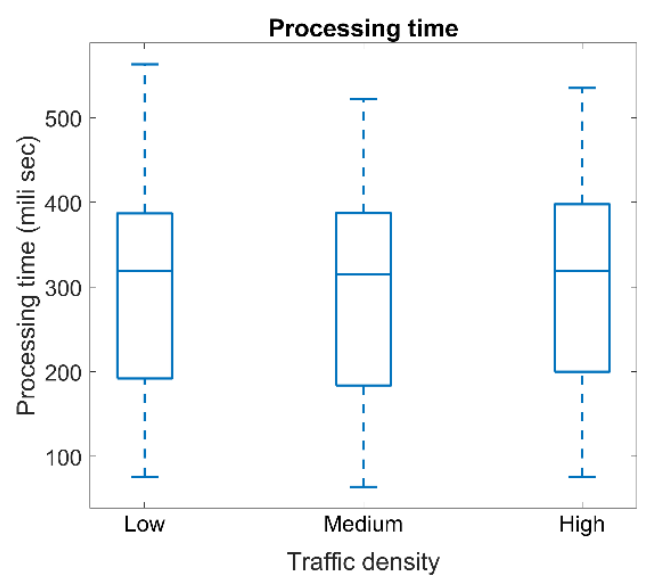

(a)

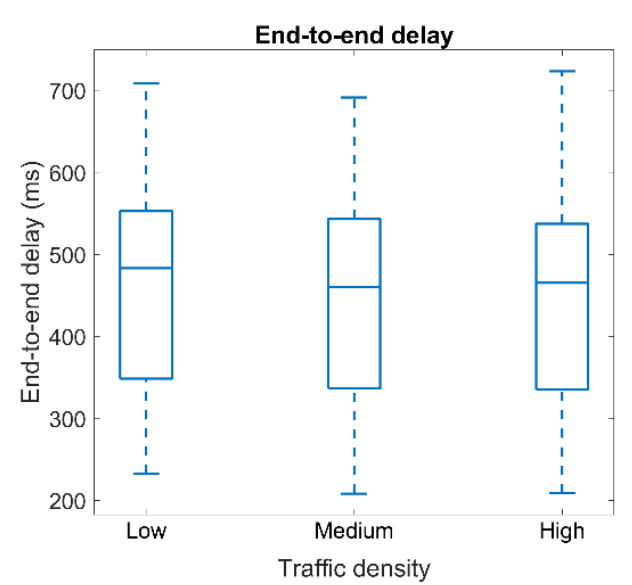

(b)

Fig. 7. Box charts of (a) processing time, and (b) end-to-end delay.

Table 3. Average (per CV) reduction of the MOEs for Serverless CloSA

\begin{tabular}{|c|c|c|c|c|c|}
\hline \multirow{2}{*}{ Delays } & \multicolumn{3}{|c|}{ Traffic Density } & \multirow{2}{*}{$\begin{array}{c}\text { Average of the } \\
\text { Three Traffic } \\
\text { Densities }\end{array}$} & \multirow[t]{2}{*}{ Allowable Delay } \\
\hline & Low & Medium & High & & \\
\hline $\begin{array}{l}\text { Average reduction in } \\
\text { stopped delay }\end{array}$ & 298 & 297 & 303 & 299 & \\
\hline $\begin{array}{l}\text { Average reduction in total } \\
\text { travel time }\end{array}$ & 463 & 447 & 446 & 452 & $<1000 \mathrm{~ms}$ \\
\hline
\end{tabular}

\section{CONCLUSION}

458 In this paper, we develop a highly scalable serverless cloud computing architecture using Amazon

459 Web Services (AWS) to support the requirements of real-time CV mobility applications. Then, we 460 develop an optimization-based real-time CV speed advisory algorithm, i.e., Serverless CloSA, which is 461 deployable using the serverless cloud-based architecture that we developed. The Serverless CloSA assists 462 CVs to pass through a signalized corridor with speed advisories that can help reduce the stopped delay 463 experienced by these CVs at the signalized intersections of that corridor. We conduct case studies for a 464 signalized corridor for three different roadway traffic conditions (low, medium, and high-density roadway 
traffic) with a cloud-in-the-loop simulation testbed using AWS and Simulation of Urban Mobility

466 (SUMO), which is an open-source microscopic roadway traffic simulator, to evaluate the feasibility and

467 performance of the Serverless CloSA at a system level. Based on the evaluation results, we conclude that

468 Serverless CloSA is effective in reducing the average stopped delay at the signalized intersections of a

469 corridor by $77 \%$ while reducing the risk of collision and the total travel time for the CVs through that

470 corridor when compared to the baseline "no speed advisory" scenario.

471 Generally, the state departments of transportation (DOTs) deploy transportation

472 applications based on traditional server infrastructure in their traffic management centers

473 (TMCs), which requires significant investments in computing and human resources. This study

474 shows that cloud infrastructure offers a promising alternative for addressing the computing

475 infrastructure needs for CV mobility applications. The commercial cloud-based CV mobility

476 application strategy could potentially lower the costs associated with computing equipment

477 installation, configuration, operation, and maintenance, without sacrificing any performance and

478 reliability.

\section{Authors Contribution}

482 H.-W. Deng, M.S. Salek, M. Rahman and M. Chowdhury wrote the main manuscript text with the help of 483 A. Apon and M. Shue. H.-W. Deng, M.S. Salek, M. Rahman, M. Chowdhury, A. Apon and M. Shue 484 conceived and planned the simulation experiments. H.-W. Deng and M.S. Salek carried out the 485 simulations. H.-W. Deng, M.S. Salek prepared all the figures. All authors critically reviewed the 486 manuscript and provided feedback to make the manuscript better. 
490 None.

491

492

\section{Conflict of Interest}

494 None.

495

496

497 REFERENCES

498 1. Deka L, Chowdhury M (2018) Transportation Cyber-Physical Systems, 1st ed. Elsevier

499 2. Omoniwa B, Hussain R, Javed MA, et al (2019) Fog/Edge Computing-Based IoT (FECIoT):

$500 \quad$ Architecture, Applications, and Research Issues. IEEE Internet of Things Journal 6:4118-4149.

$501 \quad$ https://doi.org/10.1109/JIOT.2018.2875544

502 3. Xu Z, Li X, Zhao X, et al (2017) DSRC versus 4G-LTE for Connected Vehicle Applications: A 503 Study on Field Experiments of Vehicular Communication Performance. In: Journal of Advanced 504 Transportation. https://www.hindawi.com/journals/jat/2017/2750452/. Accessed 19 Jan 2021

505 4. Deng H-W, Rahman M, Chowdhury M, et al (2021) Commercial Cloud Computing for Connected 506 Vehicle Applications in Transportation Cyberphysical Systems: A Case Study. IEEE Intelligent 507 Transportation Systems Magazine 13:6-19. https://doi.org/10.1109/MITS.2020.3037314 
510 6. Azure Functions Serverless Compute | Microsoft Azure. https://azure.microsoft.com/en511 us/services/functions/. Accessed 6 Oct 2020

512 7. What is Amazon EC2? - Amazon Elastic Compute Cloud. 513 https://docs.aws.amazon.com/AWSEC2/latest/WindowsGuide/concepts.html. Accessed 4 Mar 2021

514 8. Rajan RAP (2018) Serverless Architecture - A Revolution in Cloud Computing. In: 2018 Tenth 515 International Conference on Advanced Computing (ICoAC). pp 88-93

516 9. Shafiei H, Khonsari A, Mousavi P (2020) Serverless Computing: A Survey of Opportunities, $517 \quad$ Challenges and Applications

518 10. Islam M, Rahman M, Khan SM, et al (2020) Development and Performance Evaluation of a 519 Connected Vehicle Application Development Platform. Transportation Research Record 2674:537520 552. https://doi.org/10.1177/0361198120917146

521 11. Lopez PA, Behrisch M, Bieker-Walz L, et al (2018) Microscopic Traffic Simulation using SUMO. 522 In: 2018 21st International Conference on Intelligent Transportation Systems (ITSC). pp 2575-2582

523 12. Ning Z, Huang J, Wang X (2019) Vehicular Fog Computing: Enabling Real-Time Traffic 524 Management for Smart Cities. IEEE Wireless Communications 26:87-93. 525 https://doi.org/10.1109/MWC.2019.1700441

526 13. Li X, Dang Y, Aazam M, et al (2020) Energy-Efficient Computation Offloading in Vehicular Edge 527 Cloud Computing. IEEE Access 8:37632-37644. https://doi.org/10.1109/ACCESS.2020.2975310

528 14. Jin J, Ran B, Chen T, et al (2020) Cloud-based technology for connected and automated vehicle $529 \quad$ highway systems 
15. Bradaï B, Garnault A, Picron V, Gougeon P (2016) A Green Light Optimal Speed Advisor for Reduced CO2 Emissions. In: Langheim J (ed) Energy Consumption and Autonomous Driving.

16. Stebbins S, Kim J, Hickman M, Vu HL (2016) Combining model predictive intersection control Springer International Publishing, Cham, pp 141-151

17. Stebbins S, Hickman M, Kim J, Vu HL (2017) Characterising Green Light Optimal Speed Advisory

18. Suzuki H, Marumo Y (2018) A New Approach to Green Light Optimal Speed Advisory (GLOSA) Systems for High-Density Traffic Flowe. In: 2018 21st International Conference on Intelligent Transportation Systems (ITSC). pp 362-367

19. Pariota L, Costanzo LD, Coppola A, et al (2019) Green Light Optimal Speed Advisory: a C-ITS to

20. Zhang Z, Zou Y, Zhang X, Zhang T (2020) Green Light Optimal Speed Advisory System Designed for Electric Vehicles Considering Queuing Effect and Driver's Speed Tracking Error. IEEE Access

21. Zhao W, Ngoduy D, Shepherd S, et al (2018) A platoon based cooperative eco-driving model for mixed automated and human-driven vehicles at a signalised intersection. Transportation Research 
552 22. Serverless Computing - Amazon Web Services. In: Amazon Web Services, Inc. 553 https://aws.amazon.com/products/databases/. Accessed 29 Mar 2021

554 23. What Is Amazon DynamoDB? - Amazon DynamoDB.

555 https://docs.aws.amazon.com/amazondynamodb/latest/developerguide/Introduction.html. Accessed $556 \quad 6$ Oct 2020

557 24. Amazon Kinesis Data Streams - Data Streaming Service - Amazon Web Services. In: Amazon Web 558 Services, Inc. https://aws.amazon.com/kinesis/data-streams/. Accessed 26 Oct 2020

559 25. Fehr W (2014) Southeast Michigan Test Bed: 2014 Concept of Operations

560 26. Park B (Brian), Schneeberger JD (2003) Microscopic Simulation Model Calibration and Validation:

561 Case Study of VISSIM Simulation Model for a Coordinated Actuated Signal System.

562 Transportation Research Record 1856:185-192. https://doi.org/10.3141/1856-20

563 27. Mahmod M, Arem B van, Pueboobpaphan R, Lange R de (2013) Reducing local traffic emissions at 564 urban intersection using ITS countermeasures. IET Intelligent Transport Systems 7:78-86. 565 https://doi.org/10.1049/iet-its.2011.0222

566 28. CVXOPT. https://cvxopt.org/. Accessed 19 Jan 2021

567 29. Wegener A, Piórkowski M, Raya M, et al (2008) TraCI: an interface for coupling road traffic and 568 network simulators. In: Proceedings of the 11th communications and networking simulation 569 symposium. Association for Computing Machinery, New York, NY, USA, pp 155-163

570 30. Chowdhury M, Rahman M, Rayamajhi A, et al (2018) Lessons Learned from the Real-World 571 Deployment of a Connected Vehicle Testbed. Transportation Research Record 2672:10-23. $572 \quad$ https://doi.org/10.1177/0361198118799034 
573 31. Flow Definitions. In: Shortest or Optimal Path Routing - SUMO Documentation.

574 https://sumo.dlr.de/docs/Demand/Shortest_or_Optimal_Path_Routing.html\#flow_definitions.

$575 \quad$ Accessed 4 Mar 2021

576 32. Manual HC (2010) HCM2010. Transportation Research Board, National Research Council, $577 \quad$ Washington, DC 1207

578 33. Shi X, Wong YD, Li MZF, Chai C (2018) Key risk indicators for accident assessment conditioned 579 on pre-crash vehicle trajectory. Accident Analysis \& Prevention 117:346-356. 580 https://doi.org/10.1016/j.aap.2018.05.007

581 34. Minderhoud MM, Bovy PHL (2001) Extended time-to-collision measures for road traffic safety 582 assessment. Accident Analysis \& Prevention 33:89-97. https://doi.org/10.1016/S0001$583 \quad 4575(00) 00019-1$

584 35. Eckhoff D, Halmos B, German R (2013) Potentials and limitations of Green Light Optimal Speed 585 Advisory systems. In: 2013 IEEE Vehicular Networking Conference. pp 103-110 